\title{
Performance of HFGFlash at CMS
}

\author{
Rahmat Rahmat \\ University of Mississippi \\ E-mail: rrahmat@phy.olemiss.edu
}

\begin{abstract}
We report the performance of HF calorimeter and some details about the PMT window hit events. The new 4-anode PMTs will be installed during the 2013 shutdown period. We have developed HF GFlash, a very fast simulation of electromagnetic showers using parameterizations of the profiles in Hadronic Forward Calorimeter. HF GFlash has good agreement to $7 \mathrm{TeV}$ Collision Data and previous Test Beam results. In addition to good agreement with Data and previous Test Beam results, HF GFlash can simulate about 10000 times faster than Geant4.
\end{abstract}

\section{Introduction}

We have developed HF GFlash, a very fast simulation of electromagnetic showers using parameterizations of the profiles in Hadronic Forward Calorimeter. HF GFlash has good agreement to $7 \mathrm{TeV}$ Collision Data and previous Test Beam results. Previous HF simulation (based on Shower Library) in CMS Collaboration has no ability to simulate PMT Window Noise and other noises. The worst part of the previous simulation that it was created using only discrete energy bins $(10 \mathrm{GeV}, 20 \mathrm{GeV}, 30 \mathrm{GeV}, 40 \mathrm{GeV}, 50 \mathrm{GeV}$, etc. not continuous) that limit its precision significantly. The HF Shower Library has another problem because it deletes particles that enter HF Detector immediately and replace them with Shower Library that has very limited statistics. Fortunately, we have developed HF GFlash, a fast simulation of electromagnetic showers using parameterizations of the profiles in Hadronic Forward Calorimeter. HF GFlash solves almost all problems that previous HF simulation has.

\section{Theory}

The Gflash package allows the parameterization of electron and positron showers in homogeneous (for the time being) calorimeters and is based on the parameterization described by G. Grindhammer [2] . The spatial energy distribution of electromagnetic showers is given by three probability density functions (pdf),

$$
d E(\vec{r})=E f(t) d t f(r) d r f(\phi) d \phi,
$$

describing the longitudinal, radial, and azimuthal energy distributions. Here $t$ denotes the longitudinal shower depth in units of radiation length, $r$ measures the radial distance from the shower axis in Moliere units, and $\phi$ is the azimuthal angle. A gamma distribution is used for the parameterization of the longitudinal shower profile, $f(t)$. The radial distribution $f(r)$, is described by a two-component ansatz. In $\phi$, it is assumed that the energy is distributed uniformly: $f(\phi)=1 / 2 \pi$. 


\section{Performance of HF GFlash}

We simulated the computing time for 10,000 $20 \mathrm{GeV}$ electrons and Table 1 shows that HF GFlash can perform simulation faster than Shower Library.

\begin{tabular}{|l|c|c|}
\hline & Shower Library (previous MC) & HF GFlash \\
\hline Minimum Computing Time & 0.0095 & 0.0059 \\
\hline Maximum Computing Time & 3.01 & 2.81 \\
\hline Average Computing Time & 0.62 & 0.53 \\
\hline
\end{tabular}

Table 1. Comparison of computing time between HF GFlash and Shower Library for 10000 20-GeV electrons.

We also check the longitudinal shower profiles produced using HF GFlash and compare them to the longitudinal shower profiles produced using Shower Library. In Fig.1. we can see that longitudinal profiles produced by HF GFlash(CMSSW_3_11_0) and Shower Library(CMSSW_3_10_0_pre3).

Using the results of energy response ratio from Team Beam data as the reference, we can check the performance of energy response ratio of HF GFlash compared to Shower Library. From Table 2, we found that HF GFlash has better agreement to Test Beam data compared to Shower Library.

\begin{tabular}{|l|c|c|c|}
\hline & HF GFlash & Test Beam & Shower Library \\
\hline$S e_{50} / L e_{50}$ & 0.24 & 0.24 & 0.20 \\
\hline$L p_{50} / L e_{50}$ & 0.67 & 0.66 & 0.63 \\
\hline$S p_{50} / L e_{50}$ & 0.51 & 0.50 & 0.51 \\
\hline$S p_{50} / L p_{50}$ & 0.76 & 0.76 & 0.80 \\
\hline$S e_{100} / L e_{100}$ & 0.30 & 0.30 & 0.25 \\
\hline$L p_{100} / L e_{100}$ & 0.70 & 0.69 & 0.67 \\
\hline$S p_{100} / L e_{100}$ & 0.57 & 0.55 & 0.56 \\
\hline$S p_{100} / L p_{100}$ & 0.82 & 0.80 & 0.84 \\
\hline$S e_{150} / L e_{150}$ & 0.33 & 0.34 & 0.28 \\
\hline$L p_{150} / L e_{150}$ & 0.71 & 0.73 & 0.70 \\
\hline$S p_{150} / L e_{150}$ & 0.59 & 0.60 & 0.56 \\
\hline$S p_{150} / L p_{150}$ & 0.83 & 0.82 & 0.80 \\
\hline
\end{tabular}

$L e_{x}=$ Energy deposited in Long Fiber from $10000 \mathrm{x}-\mathrm{GeV}$ electrons $S e_{x}=$ Energy deposited in Short Fiber from $10000 \mathrm{x}-\mathrm{GeV}$ electrons $L p_{x}=$ Energy deposited in Long Fiber from $10000 \mathrm{x}-\mathrm{GeV}$ charged pions $S p_{x}=$ Energy deposited in Short Fiber from $10000 \mathrm{x}-\mathrm{GeV}$ charged pions

Table 2. Comparison of energy response ratio between HF GFlash, Test Beam(reference) and Shower Library using electrons and pions at $150 \mathrm{GeV}$.

For energy resolution, we found that HF GFlash can improve the resolution by $50 \%$. In this case, we define energy resolution as the difference of energy resolution observed in Test Beam data and simulation. For simulation we use HF GFlash or Shower Library, and we found HF GFlash has better resolution compared to Shower Library. 
Electromagnetic energy response of electrons is predicted to be linear and Test Beam data has shown that it is linear(within stat. error) up to $150 \mathrm{GeV}$. We have tuned HF GFlash so that it has linear energy response up to $14 \mathrm{TeV}$.

Previous MC simulation based on Shower Library can not simulate high energy particle for example, electrons with energy higher than $2 \mathrm{TeV}$. Fortunately HF GFlash can handle not only low energy particles but also high energy particles. We can prove that HF GFlash can produce nice longitudinal profiles correctly for $1 \mathrm{TeV}, 7 \mathrm{TeV}$ and $14 \mathrm{TeV}$ (see Figure 1)
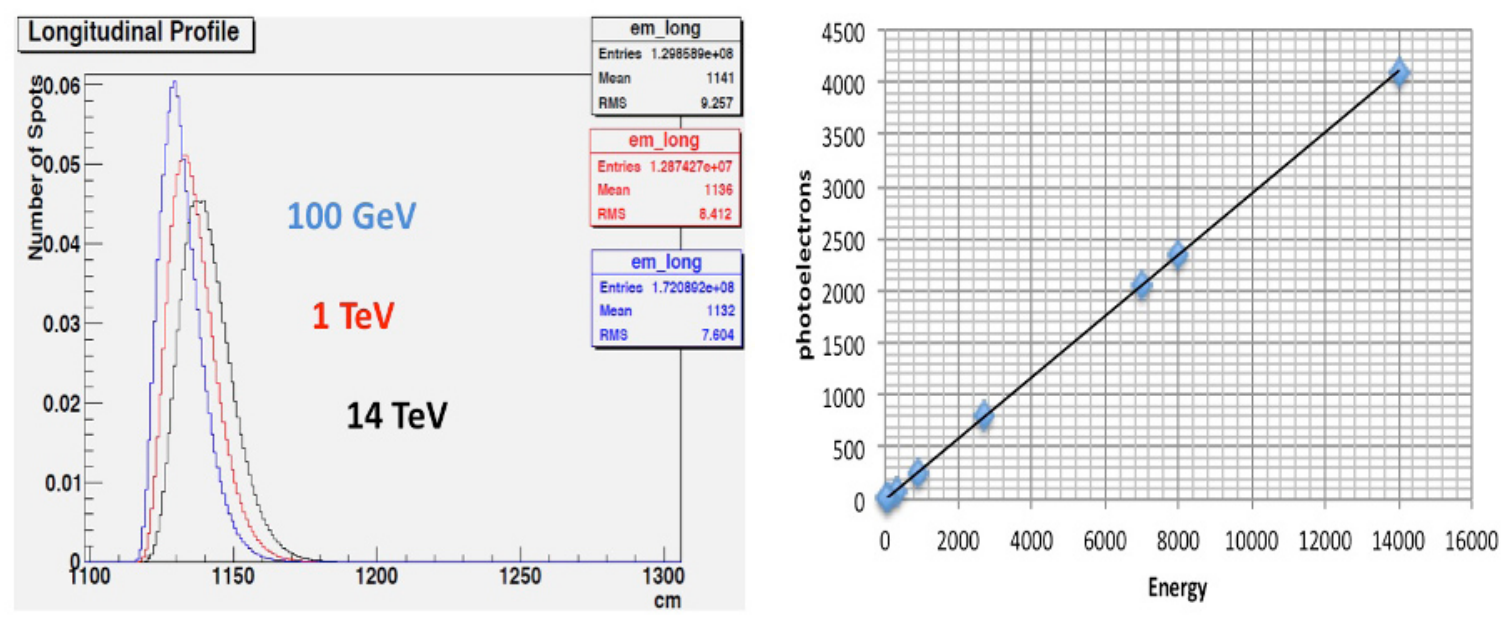

Figure 1. Left plot : Longitudinal Shower Profile produced by HF GFlash for electron with energy $100 \mathrm{GeV}$ (blue), $1 \mathrm{TeV}$ (red) and $14 \mathrm{TeV}$ (black). This plot shows the capability of HF GFlash to handle very high energy particle simulation. Right plot: HF GFlash has linear energy response for electron with energy from $50 \mathrm{GeV}$ to $14 \mathrm{TeV}$

One major breakthrough is the ability of HF GFlash to simulate PMT Window hits for the first time and previous MC simulation can not simulate PMT Window Hits. This achievement, encourage us to simulate other noises such as Fibre Bundle in HF. The early results show that inclusion of PMT Window Hits, Fibre Bundle and Jungle improve the agreement between HF GFlash Data and $7 \mathrm{TeV}$ Collision Data.

We have used HF GFlash to produce some sample datasets, for example: ttbar, $\mathrm{Z} \rightarrow \mathrm{ee}$ and MinBias. The internal memory size is very crucial and we have checked and confirmed that HF GFlash used reasonable computer memory size for physics simulation. We use the correct geometry and we have done validation of HF GFlash using CMSSW_3_10_0_pre9 (a standard CMS software combination used for analysis at the end of 2010) when HF GFlash was chosen as the default of HF simulation.

We reconstruct SimHit produced by HF GFlash to produce RecHit(reconstructed hits) that will be used for physics analysis in CMS Collaboration. We should be very careful about timing in RecHit and we see that HF GFlash gives reasonable timing information compared to certified Collision Data.

By the end of November 2010 we have collected about $36 p b^{-1}$ certified Collision Data that can be used to study RecHit(reconstructed hits) energy distribution in HF towers for Long and Short Fibres. Using MinBias generator we can simulate HF RecHit for every tower. We have made 52 comparison plots for every HF tower and we found the HF GFlash has good agreement with $36 p b^{-1}$ Certified Collision Data and we can see HF GFlash can perform better than previous MC based on Shower Library.

The crucial part of tuning is coming the next few months when we have $100 \mathrm{fb}^{-1}$ Collision 
Data from CMS Detector. At that time, we will have reasonable number of events to use $\mathrm{Z} \rightarrow \mathrm{ee}$ to tune HF GFlash with real physics process. In this analysis we will require one electron in central region as the tag and the other as the probe to check the performance of HF GFlash.

\section{HF RecHit Energy at ieta=-31}

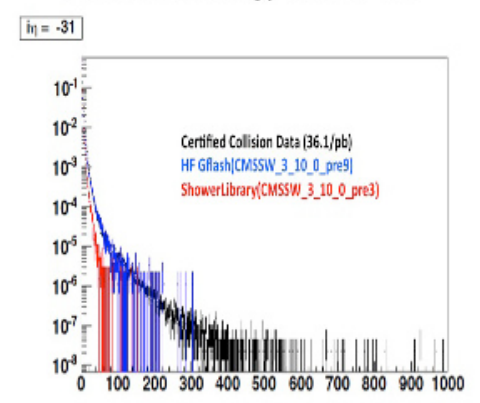

HF RecHit Energy at ieta= -33

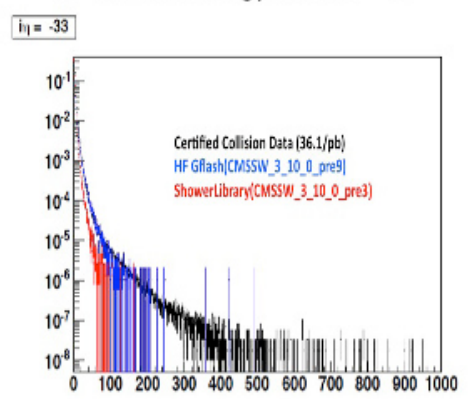

HF RecHit Energy at ieta=39

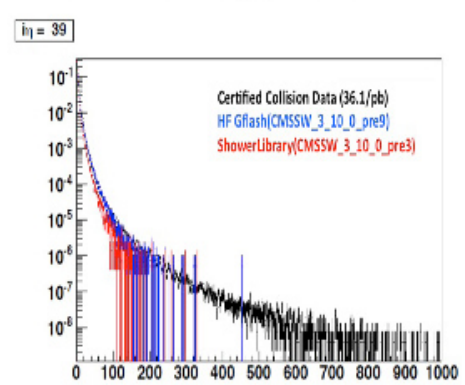

Figure 2. Recontructed hit energy at towers 31, 33 and 39 collected using HF GFlash (Blue), Shower Library (Red) and 2010 Collision Data (Black).

\section{Summary}

In summary, The HF detectors performed very well during the 2011 run period. Prior to the run, relative timing of the HF channels were improved and light guide sleeves were replaced. Operating parameters of the HF detectors were further optimized. In 2013, all the existing PMTs will be replaced with the 4-anode PMTs.

We have developed the most powerful simulation on earth that can handle very high energetic particles with better performance. Due to its better performance, CMS Collaboration has chosen HF GFlash as the standard HF Detector simulation since 2011. HF GFlash has been tested and the tests showed that it is faster and more accurate so that HF GFlash will be a very useful simulation not only for CMS Detector but also for other physics experiments, such as International Linear Collider, Muon Collider, etc.

\section{References}

[1] The CMS-HCAL Collaboration Design, performance, and calibration of CMS forward calorimeter wedges. Eur. Phys. J. C.53, 139-166(2008)

[2] G. Grindhammer, S. Peters, The Parameterized Simulation of Electromagnetic Showers in Homogeneous and Sampling Calorimeters. hep-ex/0001020 (1993).

[3] E. Longo and I. Sestili,Nucl. Instrum. Meth. 128, 283 (1975).

[4] Rossi Prentice Hall, New York (1952).

[5] G. Grindhammer, M. Rudowicz, and S. Peters, Nucl. Instrum. Meth. A290, 469 (1990).

[6] The CMS Collaboration. Technical Proposal, CERN/LHCC 94-39(1994).

[7] The CMS Collaboration, The Hadron Calorimeter Project Technical Design Report, CERN/LHCC 97-31 (1997) 